\title{
INTRAPULMONARY BRONCHOGENIC CYST: SINGLE CASE REPORT
}

\author{
How $\mathrm{SH}^{1}$, Tee HP ${ }^{2}$, AR Amran $^{3}$, ARM Fauzi ${ }^{1}$ \\ 'Department of Internal Medicine, Kulliyyah of Medicine, International Islamic University Malaysia. \\ ${ }^{2}$ Department of Internal Medicine, Hospital Tengku Ampuan Afzan, Kuantan. \\ ${ }^{3}$ Department of Radiology, Kulliyyah of Medicine, International Islamic University Malaysia.
}

\begin{abstract}
Intrapulmonary bronchogenic cyst is a rare congenital lesion originating from abnormal budding of the embryonic foregut. It is less common than mediastinal bronchogenic cyst. We describe a case of intrapulmonary bronchogenic cyst and discuss the treatment of this condition. (JUMMEC 2008; 11 (2): 86-88)
\end{abstract}

KEYWORDS: Intrapulmonary bronchogenic cyst, treatment

\section{Case Report}

A 51-year old man, who was an ex-smoker, was admitted in August 2003 with two months' history of productive cough and an episode of haemoptysis. There was no history of fever, loss of weight or appetite. He had similar complaints five years ago which were successfully treated with antibiotics. He denied having any contact with tuberculosis patients. Clinical examination was unremarkable except for minimal crepitations at the base of the left lung.

Upon admission, the patient's full blood count, renal and liver function tests were within normal limits. His erythrocyte sedimentation rate was $21 \mathrm{~mm} / \mathrm{h}$ and random blood sugar was $11.4 \mathrm{mmol} / \mathrm{l}$. Subsequently, fasting showed that his blood sugar level was normal. Three consecutive sputum samples were negative for acid-fast bacilli. Sputum for bacterial culture did not grow any organism. Chest radiograph showed a thinwalled cyst in the left lower lobe with an air-fluid level and surrounding pneumonic changes (Figure 1a).

He was treated as having lung abscess with intravenous cefotaxime (1 gm daily) and metronidazole $(500 \mathrm{mg}$ eight hourly) for ten days. Oral tranexamic acid was also given for hemoptysis. His symptoms improved after completing antibiotics for ten days and he was discharged with instructions to take oral cephalexin (250 mg four times daily) and metronidazole (400 mg twice daily) for two weeks.

However, the patient returned to the clinic a month later with worsening cough. A second chest radiograph showed minimal improvement of the air-fluid level and of the pneumonic changes (Figure 1b). Sputum culture was repeated and $H$. influenzae resistant to cefotaxime was isolated. He was given oral amoxycillinclavulanic acid (625 mg twice daily) for one month. Despite this, his cough persisted. He subsequently underwent bronchoscopic examination which showed purulent secretion within the left lower lobe bronchus. Broncho-alveolar lavage fluid grew Pseudomonas aeroginosa. Amoxycillin-clavulanic acid was replaced with perfloxacin, which was continued for two weeks. However, this failed to improve his condition. A trial of anti-tuberculous drug therapy was thought to be appropriate at this juncture. This anti-tuberculosis drug theraphy was continued for two months but this too did not alleviate the symptoms. Computerised tomography (CT) of the thorax was performed at this stage. It showed a thin-walled cyst in the left lobe with an air-fluid level and surrounding pneumonic changes (Figure $2 \mathrm{a}$ and b). There was no communication with the tracheobronchial tree. Subsequently, he underwent thoracotomy for excision of the cyst and plication of the cyst wall. Histology of the excised lesion showed changes consistent with bronchogenic cyst. He remained asymptomatic 12 months after the operation.
Correspondence:
How Soon Hin
Kulliyyah of Medicine, International Islamic University
P.O. Box 141, Kuantan
Email: how_sh @yahoo.com 
(a)

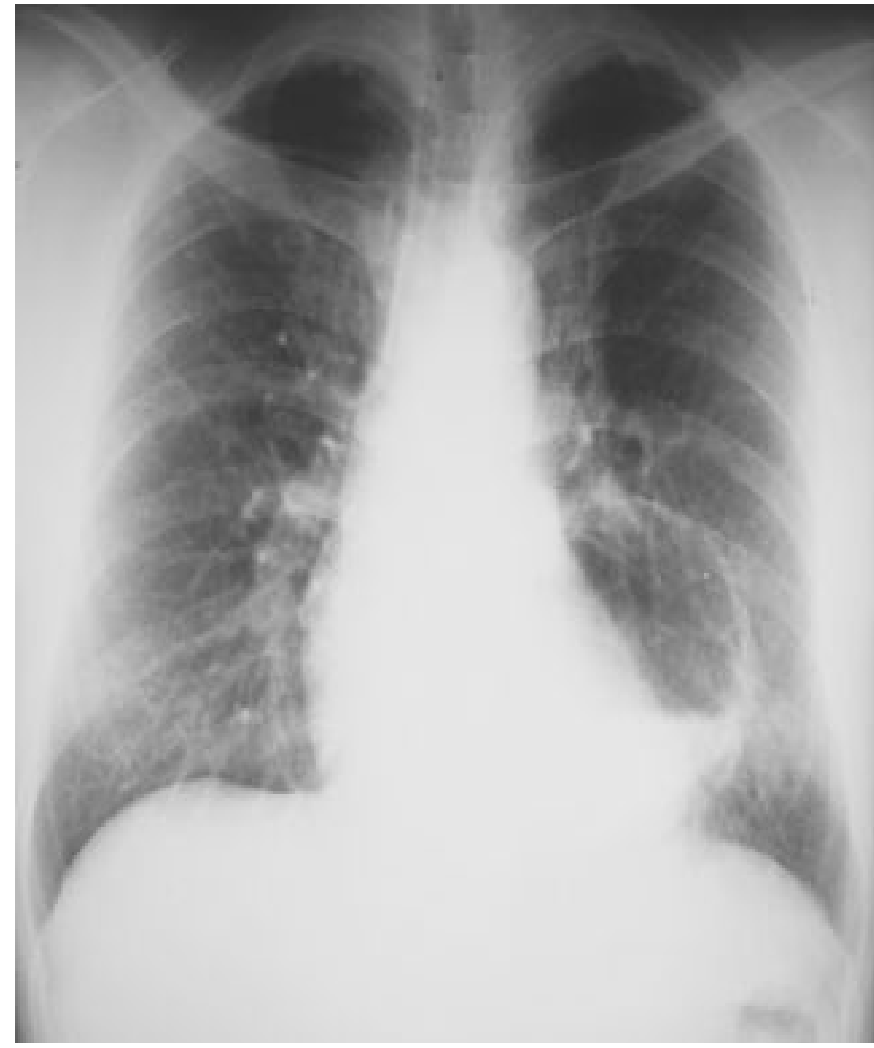

Figure 1: (a) and (b) Bronchogenic cyst. (a) Chest radiograph demonstrate a thin-walled cyst in the left lower lobe with an air-fluid level and surrounding pneumonic changes.

\section{Discussion}

Bronchogenic cysts are congenital lesions arising from the abnormal budding of the ventral foregut that occurs between the twenty-sixth and fortieth days of gestation (1). The cyst is lined by ciliated columnar or cuboidal epithelium and is surrounded by cartilage, smooth muscle, elastic tissue and mucous gland. Intrapulmonary cyst is formed if the budding occurs later in embryonic life. In contrast, early budding results in mediastinal cyst. It is very rare for this to occur in the neck, retro-peritoneal and subdiaphragmatic spaces. It can present itself at any age group and antenatal diagnosis is possible.

Intrapulmonary cyst constitutes $10-29 \%$ of all bronchogenic cysts $(1,2,3)$. Out of 11 patients reported by Mc Adam et al (1) and Yoon et al (3), six of the patients were asymptomatic on presentation. Thirty six percent had pulmonary infection and showed symptoms of fever and cough. One patient had chest pain. Hemoptysis was present in only one patient. Another patient had rupture of the infected cyst which caused pneumothorax. (b)



(b) Post-antibiotics. There was no significant change of the lesion except for the slightly decreased air-fluid level and pneumonic changes surrounding the cyst.

The appearance of intrapulmonary bronchogenic cysts on chest radiographs and CT films can be confused with acquired cysts caused by infection or other pathological etiologies such as lung abscesses, hydatid cysts, giant bullae or lobar emphysaema (1, $2,3)$. Patients were frequently treated as having lung abscess, especially in the presence of air-fluid level (2). Thickness of the cyst wall is the main differentiating point between benign bronchogenic cysts and other infection-related cysts. In chest radiographs, bronchogenic cysts are thin-walled and have smooth outline. If the cyst is infected or contained secretions, it may appear as a solid tumor or it may demonstrate an air-fluid level with surrounding pneumonic changes. The cyst contents are homogenous and do not get enhanced with radiological contrast (1). In certain cases as reported by Yoon et al, the cyst walls were not visible on both enhanced and un-enhanced CT thorax in $80 \%$ of intrapulmonary bronchogenic cysts (3). In these patients, attenuation values of the cysts on CT thorax were between 9 to $40 \mathrm{H}$. Yoon et al also noted areas of mosaic low attenuation with band-like linear attenuation in the surrounding lung parenchyma of the cysts on high resolution CT scans which represent 


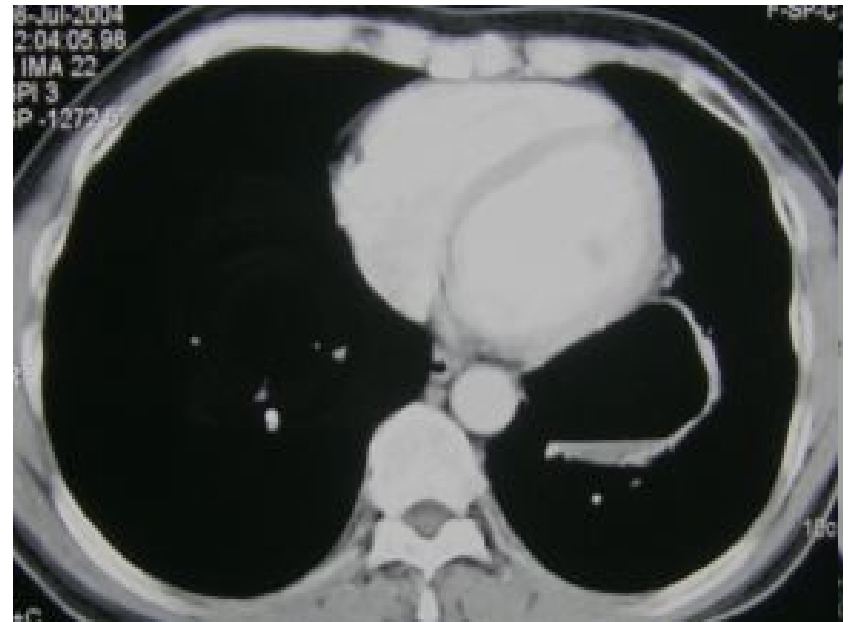

(a)

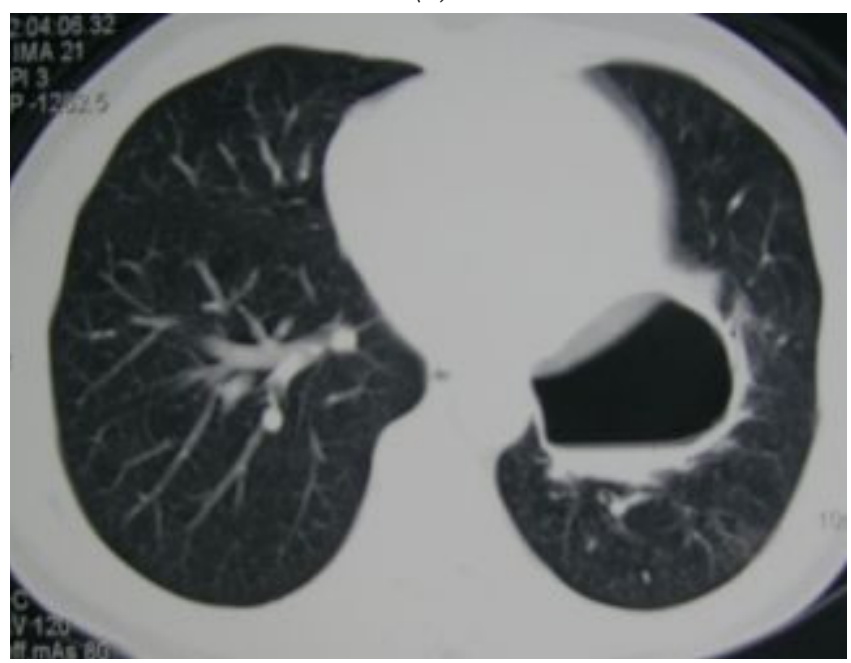

(b)

Figure 2: (a) Mediastinal and (b) Lung window. CT demonstrates a thin-walled cyst in the left lobe with an air-fluid level and surrounding pneumonic changes. There was no communication with the tracheobronchial tree.

areas of emphysema, bronchiolisation and fibrosis on histology. Air-fluid level, as seen in our case, was seen only in one of their patients. Not infrequently, the cyst may be completely filled with air (1).

Despite several radiological imaging, a definite diagnosis of bronchogenic cyst cannot be established without histological diagnosis. About half of the patients with bronchogenic cyst had other diagnosis before surgical resection (4). Cartilage on the cyst wall is an important histology to differentiate bronchogenic cyst from other acquired cyst (1). In general, there is no specific recommendation for the management of intrapulmonary bronchogenic cyst. Most of the authors advocated surgical removal of the cysts even in asymptomatic patients as complications eventually developed in the majority of the cases $(1,2,3,4)$. Some of the complications may be life threatening for example, resulting in fatal air-embolism and pneumothorax (2, 5). Furthermore, cases of complications were associated with operative difficulties and higher post-operative morbidity $(2,4)$.

In conclusion, intrapulmonary bronchogenic cyst is a rare congenital abnormality that requires surgical removal especially in patients with complications.

\section{References}

1. Mc Adam HP, Kirejczyk WM, Rosado-deChristension $\mathrm{ML}$, et al. Bronchogenic cyst: imaging features with clinical and histopathologic correlation. Radiology 2000; 217:441-446.

2. Sarper A, Arten A, Golbasi I, et al. Tex Heart Inst J 2003; 30:105-8.

3. Yoon YC, Lee KS, Kim TS, et al. Intrapulmonary bronchogenic cyst: $C T$ and pathologic findings in five adult patients. AJR Am J Roentgenol 2002; 179:167-170.

4. St-Georges R, Deslauriers J, Duranceau A, et al. Clinical spectrum of bronchogenic cysts of the mediastinum and lung in the adult. Ann Thorac Surg $1991 ;$ 52: 6-13.

5. Zaugg M, Kaplan V, Widmer U, et al. Fatal airembolism in an airplane passenger with a giant intrapulmonary bronchogenic cyst. Am J Respir Crit Care Med 1998; 157: 1686-1689. 